\title{
Suicídio entre povos indígenas: um panorama estatístico brasileiro
}

Cleane S. de Oliveira ${ }^{1}$

Francisco Lotufo Neto ${ }^{2}$

Recebido: 23/9/2002 Aceito: 18/12/2002

\section{RESUMO}

O suicídio relaciona-se etiologicamente com uma gama de fatores, que vão desde os de natureza sociológica, econômica, política, religiosa, cultural, passando pelos psicológicos e psicopatológicos, até os genéticos e os biológicos. Os autores partem de uma revisão das estatísticas mundiais, destacando os países onde a problemática é mais crítica e, em seguida, mostram uma revisão das estatísticas entre as sociedades tradicionais, detentores dos números mais alarmantes. Esses dados iniciais contextualizam a apresentação do panorama entre as populações indígenas brasileiras. O suicídio é prevalente em diversas populações, sendo relatado entre os Guarani-Apapokuva, os Urubu-Kaapor, os Paresi e os Yanomani. As taxas entre os Ticunas, com 28\% do total de óbitos entre 1994 e 1996, e os Caiowás, com taxa cerca de 40 vezes maior que a brasileira, são altamente preocupantes. Entretanto, entre os Sorowahá a situação é dramática, comunidade com 130 habitantes que tem, provavelmente, uma das maiores estatísticas mundiais, com uma taxa estimada em 1.922 por 100 mil habitantes.

Unitermos: Psiquiatria transcultural; Povos primitivos; Povos indígenas/Índios brasileiros; Taxas de suicídio.

\section{ABSTRACT}

Suicide among indigenous people: a brazilian statistical view

Suicide etiology is related to several different factors: sociological, economics, political, religious, cultural, psychological and psychopathological, and finally genetic and biological. Suicide world statistics, with emphasys where is more critical, specially the frightening figures of traditional societies, were reviewed. Data on brazilian indigenous populations were discussed. Suicide is prevalent in several indian groups, as the Guarani Apapokuva, the Urubu-Kaapor, the Paresi and the Yanomani. The Ticunas have a 28\% suicide rate among all deaths between 1994 and 1996. The Caiowá had a 40 times higher rate than the brazilian population. Among the Sorowaha the situation is dramatic: A community with 130 members has probably one of the highest world suicide rates: 1.992 for 100.000 inhabitants.

Keywords: Transcultural psychiatry; Natives; Indigenous people/ Brazilian indians; Suicide rates.

1 Médica Psiquiatra do Ambulatório de Ansiedade do Instituto de Psiquiatria do Hospital das Clínicas da Faculdade de Medicina da Universidade de São Paulo.

2 Professor Associado do Departamento de Psiquiatria da Faculdade de Medicina da Universidade de São Paulo.

Endereço para correspondência:

AMBAN - Ambulatório de Ansiedade do Instituto de Psiquiatria do HCFMUSP

Rua Ovídio Pires, s/n, São Paulo, SP - CEP 05403-010

Tel.: (0xx11) 3069-6988 Fax: (0xx11) 3085-5032

E-mail: oliveiracleane@ hotmail.com 


\section{Introdução}

O suicídio, definido pela CID-10 (X-60 a X-84) como um óbito derivado de "lesões autoprovocadas intencionalmente" por diversos métodos (CID-10, 1993), relaciona-se etiologicamente com uma gama de fatores, que vão desde os de natureza sociológica, econômica, política, religiosa, cultural, passando pelos psicológicos e psicopatológicos, até os genéticos e biológicos (Roy, 1999).

Emile Durkheim, emérito sociólogo do final do século passado, em seu estudo clássico sobre o suicídio, objetivou a compreensão de um fenômeno que era visto até então como disperso e aleatório. Concluiu que: "a taxa de suicídio varia inversamente com a integração dos grupos sociais dos quais os indivíduos fazem parte". A tipologia durkheimiana do suicídio pode ser resumida em:

- suicídio egoísta: resultado de uma individuação excessiva, com ênfase na autonomia das consciências individuais e na ausência de laços familiares ou de interações sociais significativas;

- suicídio anômico: associado ao desregramento, crises e mudanças a partir do enfraquecimento da malha social;

- suicídio altruísta: devido à subordinação dos indivíduos aos fins sociais; um ato percebido como um dever, um gesto impessoal (Durkheim, 1897).

O mesmo autor também achava que deveria haver uma correlação positiva entre o suicídio e o desemprego ou o descenso social (Durkheim, 1893), comprovada em estudos posteriores (Bloor, 1980), provavelmente, devido a uma interação entre as circunstâncias socioeconômicas e uma vulnerabilidade psicológica individual (Blumenthal, 1990).

Ainda entre as variáveis sociais, contribuem os conflitos familiares e a violência doméstica. Na Índia, $12 \%$ de todos os casos devem-se a brigas com os cônjuges ou sogro(a)s, sendo o principal fator desencadeante entre as mulheres (responsável por $45,7 \%$ dos casos) (National Crime Records Bureau, 1992).

A violência coletiva, através das guerras ou de outras perturbações sociais, pode ter diferentes efeitos sobre as taxas, até mesmo paradoxais, conforme o cenário estudado. Durante a Segunda Guerra Mundial, por exemplo, houve decréscimo dos números na população da Inglaterra, mas aumento no Japão (Desjarlais et al., 1997).

Influenciar uma decisão política ou mostrar à sociedade a magnitude do comprometimento com alguma causa norteiam os suicídios políticos ou de protesto. Exemplos são abundantes ao longo da história da humanidade. Na história política do nosso país, podemos citar o antigo, mas ainda notório, suicídio do ex-presidente Getúlio Vargas, como resposta à crise de seu governo em agosto de 1954 (Silva, 1978; Lima, 1986).

A cultura pode respaldar a auto-agressão, como se observa, entre outras situações, no seppuku, que ocorre no Japão e no sati, na Índia, onde viúvas hindus se matam diante da pira funerária do marido para honrarem sua família e ao falecido (Desjarlais, 1997).

Considerações acerca dos aspectos da religiosidade também devem ser feitas. As doutrinas católica e muçulmana condenam sua prática, e estudos na Ásia Meridional constataram que as taxas são mais altas entre os hindus do que nos praticantes das duas primeiras (Raleigh, 1993).

\section{Psicopatologia do suicídio}

Com relação às teorias psicológicas, a primeira conceitualização do instinto de morte (tanatus) veio a partir de Sigmund Freud. Em Luto e Melancolia, de 1917, o psicanalista declara sua crença de que o suicídio representaria uma agressão voltada para o íntimo, contra um objeto de amor introjetado e ambivalentemente investido, além de um desejo anteriormente reprimido de matar outra pessoa (Freud, 1953). Baseado nas idéias de Freud, Karl Menninger em El Hombre Contra si Mismo concebe três componentes de hostilidade envolvidos: o desejo de matar, o desejo de ser morto e o desejo de morrer (Menninger, 1952).

Não há consenso em afirmar que uma determinada estrutura psicodinâmica ou de personalidade estejam vinculadas diretamente ao suicídio. Mas algumas fantasias são constantemente descritas pelas vítimas como desejos de ajuda, poder, vingança, controle, punição, sacrifício, fuga, renascimento ou, ainda, de reunião com os mortos (Cantor, 1976; Hendin, 1991; Beautrais et al., 1999).

A psicopatologia é de extrema importância na gênese de numerosos casos e, segundo alguns autores, em até $90 \%$ deles há um diagnóstico psiquiátrico presente ou critérios que satisfaçam retrospectivamente a um diagnóstico (Desjarlais, 1997).

Em hospitais psiquiátricos, a mortalidade por suicídio é marcadamente aumentada, cerca de 3 a 12 vezes maior que a da população geral, e vem crescendo nos últimos anos (Engberg, 1994; Casadebaig e Philippe, 1992). Acredita-se que os parentes de primeiro grau dos pacientes psiquiátricos têm um risco quatro vezes maior de cometerem o ato (Roy, 1983). 
Verifica-se que $70 \%$ das vítimas apresentavam depressão ou alcoolismo como diagnósticos psiquiátricos de eixo I, $30 \%$ a $65 \%$ delas eram portadoras de depressão primária. $\mathrm{O}$ maior risco para ambos os sexos vem justamente quando há um transtorno do humor associado (Roy, 1999). Aí a chance aumenta cerca de 30 vezes, em relação à população geral (Guze e Robins, 1970). Já o risco entre os alcoolistas é maior de 20 a 60 vezes (Murphy e Wetzel, 1990). Nos EUA, desde 1950, o abuso de substâncias também tem sido um fator importante para o aumento das taxas em menores de 30 anos (Rich et al., 1986).

Entre os esquizofrênicos, $10 \%$ a $15 \%$ acabam cometendo suicídio (Tabbane et al., 1993), a maioria durante os primeiros anos da doença. Portanto, uma vez que seu início dá-se tipicamente na adolescência ou no começo da idade adulta, os autores tendem a ser relativamente jovens. Os sintomas depressivos nesses pacientes estão, mais uma vez, estreitamente correlacionados (Roy, 1999).

Fatores biológicos, como níveis baixos de 5-HIAA no líquor, têm sido particularmente associados com tentativas violentas (Mann et al., 1989; Nordstrom et al., 1994). A influência dos componentes genéticos advém da constatação de uma concordância significativamente maior entre pares de gêmeos monozigóticos que os dizigóticos (Roy, 1991).

Nas populações étnicas nativas, a associação entre suicídio e a presença de patologias mentais pode ser encontrada na maioria, entre os poucos trabalhos já realizados.

Em 1975, Lewis relatou uma síndrome de depressão e mutismo entre os índios norte-americanos Oglala Sioux, conhecida como wacinko. O quadro era caracterizado por graus variáveis de retraimento social, medo patológico e retardo psicomotor, até o mutismo, imobilidade e, por fim, comportamento suicida.

Um estudo realizado com um grupo de índios norte-americanos de uma comunidade grande do sudoeste dos EUA encontrou uma prevalência de transtorno de estresse pós-traumático (PTSD) comparável às taxas de comunidades expostas a eventos extremos (como sobreviventes de massacres, incêndios, combates, etc.). Havia também correlação positiva entre o PTSD e outros transtornos psiquiátricos, como depressão e abuso/dependência de substâncias (Robin et al., 1997).

Outro estudo epidemiológico em índios adolescentes norte-americanos entre 9 e 15 anos, na sua maioria do grupo Qualla e Cherokee, mostrou uma correlação positiva entre um diagnóstico psiquiátrico primário e o abuso de álcool subseqüente, além da alta prevalência do consumo de tabaco, maconha e outras drogas ilícitas (Federmann et al., 1997).

A análise de uma amostra de pacientes esquimós do oeste do Alaska que haviam tentado suicídio entre 1992 e 1993 revelou que o consumo prévio de álcool estava presente em quase todos os casos. A maioria tinha um diagnóstico de depressão ou alcoolismo. Outros dados relevantes foram um precipitante psicossocial (como perda parental na infância) ou interpessoal recente, além da limitação na habilidade de verbalização da angústia (Gregory, 1994).

Outra amostra, selecionada dentre os pacientes de um serviço psiquiátrico destinado ao atendimento de nativos do Canadá (principalmente índios da etnia Cree), apresentava uma prevalência de $40 \%$ de depressão maior, $10 \%$ de alcoolismo e $10 \%$ de transtornos psicóticos (Peltz et al., 1981).

Em Taiwan, um estudo caso-controle em aborígenes, utilizando entrevistas biográficas retrospectivas para a investigação de sessenta suicídios consecutivos numa aldeia, constatou que quase todos tinham uma ou mais patologias mentais. As mais prevalentes eram depressão maior, seguida de transtornos decorrentes do uso de álcool, distimia, transtornos mentais de etiologia orgânica e esquizofrenia (Cheng, 1995).

\section{As taxas mundiais}

Em 1990, mais de 1,4 milhão de pessoas se suicidaram, o equivalente a aproximadamente $1,6 \% \mathrm{da}$ mortalidade mundial, ficando o suicídio entre as dez principais causas de morte (Banco Mundial, 1993). Se as tentativas também forem computadas, esse número torna-se de 10 a 20 vezes maior (Diekstra e Gulbinat, 1993).

Sobreposto a esses valores, há que se considerar uma importante subestimação nas estatísticas, absolutamente pouco fidedignas, por tratar-se de um ato ainda imbuído de muita estigmatização. Somente 39 dos 166 países membros das Nações Unidas publicam e fornecem seus dados oficiais, mesmo assim, com um sub-registro calculado em $30 \%$ a $200 \%$ (Diekstra, 1993).

Os índices variam muito de país para país e não têm correlação direta com seu grau de desenvolvimento ou industrialização, como mostra a tabela que se segue (Tabela 1), sendo três vezes mais freqüentes nas áreas rurais que nas urbanas (OMS, 1991).

O Sri Lanka tem a taxa mais alta do mundo na atualidade, o que tem sido correlacionado à sua história recente de violência política e social. A China também se destaca, apresentando um predomínio entre mulheres jovens (Silva, no prelo). 
Tabela 1 Taxas de suicídio (por 100 mil habitantes) segundo faixa etária. Países selecionados no período de 1986 a 1991

\begin{tabular}{lcccc}
\hline País & Ano & $\begin{array}{c}\text { Taxa } \\
\mathbf{1 5} \mathbf{~ a ~} \mathbf{2 4} \text { anos }\end{array}$ & $\begin{array}{c}\text { Taxa } \\
\mathbf{6 5} \text { a 74 anos }\end{array}$ & Total \\
\hline Sri Lanka & 62,5 & 48,6 & 33,2 \\
China & 1986 & 21,3 & 47,8 & 17,1 \\
Austrália & 1989 & 16,4 & 16,7 & 13,3 \\
Canadá & 1988 & 15,0 & 12,6 & 12,7 \\
URSS & 1990 & 13,9 & 30,4 & 21,1 \\
EUA & 1990 & 13,3 & 18,0 & 12,2 \\
Hungria & 1989 & 12,6 & 61,5 & 38,6 \\
Alemanha & 1991 & 9,9 & 23,7 & 15,8 \\
Japão & 1990 & 7,0 & 27,6 & 16,1 \\
Chile & 1991 & 6,7 & 9,6 & 5,6 \\
Argentina & 1989 & 5,2 & 19,2 & 7,1 \\
\hline
\end{tabular}

Fonte: OMS, Divisão de Saúde Mental, 1991 (OMS, 1991)

Em muitos países europeus, o suicídio já foi considerado um problema de saúde pública, principalmente na Hungria, que possui a maior cifra do continente. Um estudo multicêntrico envolvendo 16 cidades em 13 países, realizado entre 1989 e 1992, constatou que as vítimas eram, em sua maioria, jovens pertencentes a categorias sociais mais sujeitas a instabilidade e pobreza (Schmidtke, 1996).

No Japão ocorreu uma elevação significativa dos casos no pós-guerra, que ainda permanecem relativamente freqüentes. Há um contexto de aprovação cultural do suicídio ritual (o seppuku, conhecido no ocidente como harakiri), que possibilita a restauração da honra pessoal e familiar em resposta ao sofrimento de uma humilhação (Tatai e Tatai, 1991).

Nas Américas, recentemente tem-se observado um aumento da incidência, principalmente nos EUA, Chile, Argentina e Trinidade e Tobago (neste último, chegou a 15,3 por 100 mil em 1986) (OPS, 1993).

Apesar de uma tendência à relativa estabilização das taxas mundiais para a população em geral, nos últimos quinze anos a situação tornou-se muito preocupante entre os jovens. Considerando-se a faixa etária dos 15 aos 24 anos, observou-se um aumento de cerca de 2 a 3 vezes nos últimos trinta anos, tornando-se a terceira causa de mortalidade (Roy, 1999).

\section{Taxas de suicídio entre as sociedades tradicionais}

Entre todas as comunidades étnicas, os povos nativos indígenas possuem as piores estatísticas (Grossman, Milligan e Deyo, 1991). Valores muitíssimo altos, em proporções quase epidêmicas, são relatados entre algumas populações de Ilhéus no Pacífico Sul. Na ilha de Truk (Micronésia), para a faixa de 15 a 24 anos, no período de 1978 a 1987, foram 207 suicídios por 100 mil habitantes, tornando-se a primeira causa de morte entre adolescentes do sexo masculino (Youth Suicide Surveillance, 1986). Em Gainj (Papua-Nova Guiné), a taxa anual média alcançou os surpreendentes 1.200:100.000 entre as mulheres de 20 a 49 anos, no período de 1978 a 1979 (Rubinstein, 1983). Um padrão semelhante e ascendente também é descrito entre os aborígenes da Austrália, nativos de Samoa Ocidental e os maoris da Nova Zelândia (Skegg et al., 1995).

Os índios norte-americanos e os nativos do Canadá e do Alaska apresentam variabilidade entre as tribos estimada em 8 a 120 casos por ano (para 100 mil habitantes) (Shore, 1975). Nota-se um nítido aumento nos últimos anos (Dizmang et al., 1974). No período de 1980 a 1982, a taxa foi 1,7 vez maior que a da população geral dessas regiões (Manson et al., 1989) e entre os jovens dos 10 aos 24 anos chega a ser 2,8 vezes maior (May, 1987), com predominância no sexo masculino (Corin, 1995).

Desde a década de 1970, epidemias foram descritas entre os índios adolescentes norte-americanos, principalmente nas reservas de Wind River, Yakima e nas aldeias de Bethel e Kotzebue (Manson et al., 1989). Recentemente, o problema tem sido enfrentado por várias tribos, como os grupos Shoshonean (98:100.000) (Dizmang et al., 1974), Apaches (36,5:100.000), Pima, Papago e Yaqui (30:100.000) (Santora e Starkey, 1982), Pueblos (23,2:100.000) e Navajos (15,8:100.000) (OMS, 1997). Em alguns nativos no Canadá, a média chega a 267:100.000 (Fox, Manitowabi e Ward, 1984).

$\mathrm{Na}$ história da etnologia indígena da América do Sul, quase não se encontram registros contabilizados. Muitos casos foram relatados para os Aguaruna, da família lingüística Jívaro, que ocupam regiões da Amazônia Peruana (Brown, 1986). 


\section{As estatísticas brasileiras e o caso Sorowahá}

No Brasil, apesar da pouca confiabilidade dos dados e dos parcos recursos destinados à sistematização de uma boa coleta, a Fundação Nacional de Saúde computou 6.594 casos no território nacional, em 1995, sendo a maior taxa entre a população de 20 a 39 anos (FNS, 1988). Mas, segundo Mello Jorge (1995), o suicídio é a mais passível de subestimação entre as causas externas.

Somente no município de São Paulo foram 5,2:100.000 no ano de 1991. Chama atenção a sua ocorrência entre adolescentes e o pico entre 20 e 24 anos (Gawryszewski, 1995). Mas essa taxa também deve estar sujeita a sub-registro, principalmente das mortes por envenenamento (Allebeck et al., 1991).

$\mathrm{Na}$ etnologia brasileira, parece que o hábito já era comum entre os Guarani-Apapokuva e os Urubu-Kaapor em meados deste século. Mas, apesar das descrições de casos esparsos posteriormente, em vários grupos, como os Paresi, os Yanomami (dal Poz, 1999) e os Ticuna (nestes últimos, com uma taxa de $28 \%$ do total de óbitos entre 1994 e 1996) (Erthal, 1998), a questão somente veio à tona após o destaque dado pela imprensa leiga à "epidemia" ocorrida entre os Guarani, nas proximidades do município de Dourados (MS), a partir da década de 1980.

No seu ano mais crítico, em 1995, foram 55 casos fatais para uma população de 25,5 mil habitantes (CIMI, 1996). Esse valor equivaleria a uma taxa de $215,7: 100.000$, cerca de 40 vezes a média brasileira. O caráter seqüencial e, novamente, a predominância entre os adolescentes eram chamativos (Cassorla e Smeke, 1994; Levcovitz, 1998).

A situação mais crítica já descrita está entre o grupo indígena Sorowahá, do ramo lingüístico Arawá (ou aruaque). Tem uma população estimada em 130 habitantes, pelo último censo brasileiro de 1997, e ocupa uma área demarcada próximo ao município de Tapauá (AM), na região do Médio Purus, importante rio da Bacia Hidrográfica Amazônica (IBGE, 1997). Vivem ainda da agricultura de subsistência, caça, pesca e coleta (Kroemer, 1985).

Afastados das principais vias de navegação, esses índios mantiveram seu isolamento até o fim da década de 1970, quando foram localizados pelas notícias de conflitos com sorveiros (caboclos que vivem do extrativismo da sorva, fruto babáceo comestível da sorveira), que haviam invadido seu território.

Desde os primeiros contatos, em 1980, os indigenistas depararam-se com tentativas de suicídio freqüentes, através do envenenamento pela ingesta do sumo da raíz de um tipo de timbó (o konaha - provavelmente uma leguminosa do gênero lonchocarpus, cujo princípio ativo é a rotenona).

Através de um levantamento genealógico, que remontou até seis gerações passadas, foram reportados 122 casos (75 homens e 47 mulheres) anteriores à data do primeiro contato, em 1979, e 38 casos (18 homens e 20 mulheres) de 1980 a 1995. Em todos os períodos, os indivíduos eram, majoritariamente, jovens entre $14 \mathrm{e}$ 28 anos (Tabela 2).

Tabela 2 Taxas de suicídio (por gênero e faixa etária) Comunidade Sorowahá

\begin{tabular}{lcccc}
\hline & \multicolumn{2}{c}{ Até 1979 } & \multicolumn{2}{c}{ De 1980 a 1985 } \\
& Total & $\begin{array}{c}\text { Casos entre } \\
\text { 14 e 28 anos }\end{array}$ & Total & $\begin{array}{c}\text { Casos entre } \\
\text { 14 e 28 anos }\end{array}$ \\
\hline Homens & 75 & 53 & 18 & 15 \\
Mulheres & 47 & 29 & 20 & 11 \\
Total & 122 & 82 & 38 & 26 \\
\hline
\end{tabular}

Amostras de extensão reduzida e eventos de baixa freqüência impõem cautela no manuseio de dados demográficos nas sociedades de pequena escala (Adams e Price, 1994). Mas, tomando-se a população Sorowahá, com uma média estimada em 123,6 pessoas para o último período, de 1980 a 1995 , as cifras corresponderiam a uma projeção aritmética de 1.922 casos para 100 mil habitantes, quase 10 vezes o valor estimado para os Guarani (dal Poz, 1999).

Mesmo com números tão alarmantes, caminhando talvez para o auto-extermínio, esse grupo foi objeto apenas de estudos preliminares no campo da etnografia e da lingüística, de teor mais descritivo (Kroemer, 1985; 1989; 1994; Silva, 1994; Suzuki, 1995; Fank e Porta, 1996).

\section{Conclusão}

Os estudos realizados entre as populações de nativos em vários lugares do mundo (no Brasil esse tipo de trabalho ainda tem sido escasso) apontam invariavelmente para a importância dos fatores de risco vinculados à psicopatologia, além daqueles ligados aos aspectos socioeconômicos e culturais.

Na medida em que o suicídio pode ser explicado como expressão da psicopatologia, suscetível à intervenção, estaríamos diante da ocorrência de mortes potencialmente evitáveis. Uma revisão recente dos estudos sobre suicídio sugere que a melhoria do diagnóstico e do tratamento das doenças mentais seria uma excelente estratégia para a prevenção de novos casos na população geral (Henriksson et al., 1993; Mortensen, 1999). 
Estamos então diante de uma problemática na qual se salienta a necessidade da disposição de programas preventivos e de tratamento, elaborados por profissionais de saúde mental (Desjarlais, 1997).

Contudo, há que se considerar sempre a especificidade que cada grupo impõe para si. Daí a necessidade de mais pesquisas para a produção de dados particularizados na área da suicidologia, visando relevância cultural e sociológica na fundamentação das estratégias de prevenção e assistência em saúde mental (Santora e Starkey, 1982).

\section{Referências bibliográficas}

Adams, K.; Price, D. - The Demography of Small-Scale Societies: Case Studies from Lowland South America. South American Indian Studies 4: 1994.

Allebeck, P. et al. - Causes of Death in a Cohort of 50465 Young Men - Validity of Recorded Suicide as Underlying Cause of Death. Scan J Soc Med 19: 242-7, 1991.

ANôNImo. - Diretrizes Éticas Internacionais para Pesquisas Biomédicas Envolvendo Seres Humanos. Bioética 3: 95-136, 1995.

Association of Canadian Universities for Northern Studies - Ethical Principles for the Conduct of Research in the North. ACUNS Occasional Publication 7. Deposited at the National Library of Canada. Ottawa, 1982.

Banco Mundial - Informe Sobre el Desarrollo Mundial 1993: Invertir em Salud. Oxford University Press, New York, 1993.

Beautrais, A.L.; Joyce, P.K.; Mulder, R.T. - Personality Traits And Cognitive Styles as Risk Factors for Serious Suicide Attempts Among Young Peolple. Suicide Life Treat Behav 29: 37-47, 1999 .

Bloor, M. - Relationships Between Unenployment Rates and Suicide Rates in Eight Countries 1962-1976. Psychological Reports 47: 1095-101, 1980.

Blumenthal, S.K.; Kupfer, D. (eds). - Suicide Over the Life Cycle: Risk Factors, Assessment and Treatment of Suicidal Patients. American Psychiatric Press, Washington, 1990.

Brown, M.F. - Power, Gender and the Social Meaning of Aguaruna Suicide. Man 21(2): 311-28, 1986.

Canino, GJ.; Rubio-Stipec, M.; Bravo, M. - La Classificacion Diagnostica Psiquiatrica en Estudios Epidemiologicos Tranculturales. Acta Psiq Amér Lat 34(3): 251-259, 1988.

CAntor, P. - Personality Characteristics Found Among Youthful Female Suicide Attempters. J Abnorm Psychol 85(3): 324-9, 1976.

Casadebaig, P.; Philippe, A. - Mortality Due to Suicide, Accidents and Undertermined Causes in Hospitalized Mental Patients 19681982. Rev Epidemiol Sante Publique 40: 126-35, 1992.

Cassorla, R.M.S.; Smeke, E.L.M. - Autodestruição Humana. Cad Saúde Públ 10(1): 61-73, 1994.

Cheng, A.T.A. - Mental Illness and Suicide. Arch Gen Psychiatry 52: 594-603, 1995.

Cimi-Conselho Indigenista Missionário - A Violência Contra os Povos Indígenas no Brasil: 1994-1995. Brasília, pp. 36-38, 1996.

Conselho Nacional de Saúde (CNS) - Resolução n 01/88: As Normas de Pesquisa em Saúde. Bioética 3:137-54, 1995.

Control Council Law 1949. - Cógido de Nuremberg. Tribunal Internacional de Nuremberg, 1947. Control Council Law 10(2): 181-2, 1949
Corin, E. - The Cultural Frame: context and Meaning in the Construction of Health. In: (eds.) Society and Health. Oxford University Press, New York, 1995.

dal Poz, J.D. - Crônica de uma Morte Anunciada: Do Suicídio Entre os Sorowahá. Rev Antropol 43(1): 89-144, 1999.

Desjarlais, R.; Eisenberg, L; Good, B.; Kleinman, A. - Salud Mental en el Mundo: Problemas y Prioridades en Poblaciones de Bajos Ingresos. Organización Panamericana de la Salud, 1997.

Diekstra, R.F.W. - The Epidemiology of Suicide and Parasuicide. Acta Psychiatr Scand 371: 9-20, 1993.

Diekstra, R.F.W.; Gulbinat, W. - The Epidemiology of Suicidal Behavior: a Review of Three Continents. World Health Statistics Quarterly 46: 52-68, 1993.

Dizmang, L.H.; Watson, J.; MAy, P.A.; Boop, J. - Adolescent Suicide at an Indian Reservation. Amer J Orthopsychiatry 44(1): 43-9, 1974.

Durkheim, E. - A Divisão do Trabalho Social. Vol.II, Ed. Presença/ Martins Fontes, Lisboa, 1977 [1893].

Durkheim, E. - Le Suicide: Étude de Sociologie. Quadrige/PUF, Paris, 1993 [1897].

Engberg, M. - Mortality and Suicide Rates of Involuntarily Comitted Patient. Acta Psychiatr Scand 89: 35-40, 1994.

ERThal, R.M.C. - O Suicídio Ticuna na Região do Alto Solimões - AM. Dissertação para Obtenção do Título de Doutorado pela Escola Nacional de Saúde Pública - FIOCRUZ. Rio de Janeiro, 1998.

FAnK, J.; Porta, E. - A Vida Social e Econômica dos Sorowahá. OPAN/CIMI. Cuiabá, 1996.

FAnk, J.; Porta, E. - Mitos e Histórias dos Sorowahá. OPAN/CIMI. Cuiabá, 1996.

FAnk, J.; PorTA, E. - Vocabulário da Língua Sorowahá. OPAN/CIMI. Cuiabá, 1996.

Fox, J.; Manitowabi, D.; Ward, J.A. - An Indian Community With a High Suicide Rate - 5 years after. Can J Psychiatry 29: 425-7, 1984.

Federmann, E.B. et al. - Development of Substance Use and Psychiatric Comorbidity in an Epidemiologic Study of White and American Indian Young Adolescents - The Great Smoky Mountains Study. Drug and Alcohol Dependence 44: 69-78, 1997.

Freud, S. - Standard Edition of the Complete Psychological Works of Sigmund Freud. Hogarth Press. London, pp.1066, 1953.

FundaÇão NaCional de SAÚde - Mortalidade - Brasil - 1995. CENEPI/ Ministério da Saúde. Brasília, pp. 34, 1998.

Gawryszewski, V.P. - A Mortalidade por Causas Externas no Município de São Paulo, 1991. Tese de Dissertação para Obtenção do Título de Mestre, Apresentada ao Departamento de Epidemiologia da Faculdade de Saúde Pública da Universidade de São Paulo. São Paulo, 1995.

Gregory, R.J. - Grief and Loss Among Eskimos Attempting Suicide in Western Alaska. Am J Psychiatry 151(12): 1815-6, 1994.

Grossman, D.C.; Milligan, B.C.; Deyo, R.A. - Risk Factors for Suicide Attempts Among the Navajo Adolescents. American J Public Health 81(7): 870-4, 1991.

GuimarÃEs, P.M. - Legislação Indigenista Brasileira. Edições Loyola. São Paulo, 1989.

Guze, S.B.; Robins, E. - Suicide Among Primary Affective Disorder. Br J Pychiatry 117: 437-8, 1970.

Hendin, H. - Psychodynamics of Suicide, with Particular Reference to the Young. Am J Psychiatry 148(9): 1150-8, 1991.

Henriksson, M.M. et al. - Mental Disorders and Comorbidity in Suicide. Am J Psychiatry 150: 935-40, 1993.

IBGE-Instituto Brasileiro de Geografia e Estatística. - Anuário Estatístico do Brasil 1997. Vol. 57, pp. 1-174, 1997. 
Johnson, P.L. - When Dying is Better than Living: Female Suicide Among the Gainj of Papua New Guinea. Ethnology 20: 325-34, 1981.

Kortman, F. - Problems in Comunication in Transcultural Psychiatry. Acta Psychiatr Scand 75(28): 563-70, 1987.

Kroemer, G. - Cuxiuara, o Purus dos indígenas: Ensaio Etno-Histórico Sobre os Índios do Médio Purus. Edições Loyola. São Paulo, pp. 144-8, 1985.

Kroemer, G. - A Caminho das Malocas Zuruahá. Ed. Loyola. São Paulo, 1989.

Kroemer, G. - Kunahã Made, O Povo do Veneno: Sociedade e Cultura do Povo Zuruahá. Ed. Mensageiro, Belém, 1994

Levcovitz, S. - Kandire: O Paraíso Terreal. O Suicídio entre os Índios Guaranis do Brasil. Ed Espaço e Tempo/ Te Corá. Rio de Janeiro, 1998.

Lewis, T.H. - A Syndrome of Depression and Mutism in the Oglala Sioux. Am J Psychiatry 132(7): 753-5, 1975.

Lewis-Fernández, R.; Kleina Man, A. - Cultural PsychiatryTheoretical, Clinical, and Research Issues. Psychiatr Clin North Am 18(3): 433-48, 1995

Lima, V.R. - Getúlio, Uma História Oral. Ed. Record, Rio de Janeiro, 1986.

Mann, J. et al. - Evidence for the 5-HT Hypothesis of Suicide: A Review of Post-Mortem Studies. Br J Psychiatry 155: 7, 1989.

Manson, S.M.; Beals, J.; Dick, R.W.; Duclos, C. - Risk Factors for Suicide Among Indian Adolescents at a Boarding School. Pub Health Reports 104(6): 609-14, 1989.

MAY, P.A. - Suicide Among American Indian Youth: a Look at the Issues. Children Today 16(4): 22-5, 1987.

Mello Jorge, M.H.P. - Mortalidade por Causas Violentas no Município de São Paulo. Dissertação para Obtenção do Título de Doutor, Apresentada a Faculdade de Saúde Pública da Universidade de São Paulo. São Paulo, 1995.

Menninger, K. - Los motivos (Cap. II). In: El Hombre Contra Si Mismo. Editorial Losada. Buenos Aires, pp. 29-86, 1952.

Mortensen, P.B. - Can Suicide Research Lead to Suicide Prevention? Acta Psychiatr Scand 99: 397-8, 1999.

Murphy, GE.; Wetzel, R.D. - The Lifetime Risk of Suicide in Alcoholism. Arch Gen Psychiatry 47: 383-92, 1990.

National Crime Records Bureau. Governo da Índia, 1992.

National Health and Research Council - Guidelines on Ethical Matters in Aboriginal and Torres Strait islanders helth research. Camberra, 1991.

Nordstrom, P. et al. - CSF 5-HIAA Predicts Suicide Risk After Attempt Suicide. Suicide Life Threat Behav 24: 1, 1994

Organização Mundial de Saúde CID-Classificação Estatística Internacional de Doenças e Problemas Relacionados à Saúde. Décima Revisão. Centro Colaborador da OMS para a Classificação de Doenças em Português. Edusp. São Paulo, 1993

Organização Mundial de Saúde - Anuário Estatístico 1991 (para 1989). Organização Mundial de Saúde. Genebra, 1991.

Organização Panamericana de Saúde - In: Yunes, J. Mortalidad por Causas Violentas em la región de las Américas. Bulletin of PAHO 27(2), 1993.
Patel, V.; Simunyu, E.; Gwanzura, F. et al. - The Shona Sympon Questionnaire: The Development of an Indigenous Measure of Common Mental Disorders in Harare. Acta Psychiatr Scand 95: 469-75, 1997

Peltz, M. et al. - Clinical Data From a Psychiatric Service to a Group of Native People. Can J Psychiatry 26: 345-8, 1981.

Raleigh, S.B. - Suicide and Asian Religions (letter). Br J Psychiatry 162: 124,1993

Rich, C.L. et al. - San Diego Suicide Study: Young vs. Old Subjects. Arch Gen Psychiatry 43(6): 577-82, 1986

Robin, R.W. - Prevalence and Characteristics of Trauma and Posstraumatic Stress Disorder in a Southwestern American Indian Community. Am J Psychiatry 154: 1582-88, 1997.

Room, R.; JAnCA, A; BennetT, L.A et al. - WHO Cross-Cultural Applicability Research on Diagnosis and Assessment of Substance Use Disorders: An Overview of Methods and Selectd Results. Addiction 91(2): 199-20, 1996.

Roy, A. - Family History of Suicide. Arch Gen Psychiatry 40: 971, 1983.

Roy, A. et al. - Suicide in Twins. Arch Gen Psychiatry 48: 29, 1991.

RoY, A. - Emergências Psiquiátricas. In: KAPLAN, H.I.; SADOCK, B.J. (eds). Tratado de Psiquiatria. Vol. 2, 6a ed., Artmed. Porto Alegre, pp. 185, 1999.

RubinsteIn, D.H. - Epidemic Suicide Among Micronesian Adolescents. Soc Scien and Medicine 17: 657-65, 1983.

SAntora, D.; Starkey, P. - Research Studies in American Indian Suicide. JNMHS 20: 25-9, 1982.

Schmidtke, A. et al. - Attempted Suicide in Europe: Rates, Trends and Sociodemographic Characteristics of Suicide Attempters During The Period 1989-1992. Results of the WHO/EURO Multicentre Study on Parasuicide. Acta Psychiatr Scand 93: 327-38, 1996.

Shore, J.H. - American Indian Suicide - Fact and Fantasy. Psychiatry 38: 86-91, 1975.

SiLva, H.R. - 1954: Um Tiro no Coração - O Ciclo de Vargas. Vol. XVI. Ed. Civilização Brasileira. Rio de Janeiro, 1978.

Silva, M.L. - Um Estudo Sobre a Sociedade Zuruahá (versão provisória), mimeo., p.12, 1994.

Skegg, K.; Cox, B.; Broughton, J. - Suicide Among New Zealand Maori: Is History Repeating itself? Acta Pysichiatr Scand 92: 453-59, 1995

Suzuki, M. - Esboço Preliminar da Fonologia Zuruahá. In: Wetzels, L. (org.). Estudos Fonológicos das Línguas Indígenas Brasileiras. Ed. UFRJ. Rio de Janeiro, pp. 341-78, 1995.

Tabbane, K; Joober, R; Spadone, C et al. - Mortality and Cause of Death in Schizophrenia: Review of the Literature. Encephale 19: 23-8, 1993.

Tatai, K.; Tatai, K. - Suicide in the Elderly: A Report From Japan. Crisis 12(2): 40-3, 1991.

WING, J.K.; B ABOR, T.; BRUGHA, J. et al. - SCAN Schedules for Clinical Assessment in Neuropsychiatry. Arch Gen Psychiatry 47: 58993, 1990.

World Medical Association - Declaration of Helsinki, 1964. BMJ 2: $177,1964$.

Youth Suicide Surveillance - Centers for Disease Control. Atlanta, GA, 1986. 